\title{
Hölder Continuous Solutions to Complex Hessian Equations
}

\author{
Ngoc Cuong Nguyen
}

Received: 24 January 2013 / Accepted: 4 March 2014 / Published online: 8 April 2014

(C) Springer Science+Business Media Dordrecht 2014

\begin{abstract}
We prove the Hölder continuity of the solution to complex Hessian equation with the right hand side in $L^{p}, p>\frac{n}{m}, 1<m<n$, in a $m$-strongly pseudoconvex domain in $\mathbb{C}^{n}$ under some additional conditions on the density near the boundary and on the boundary data.
\end{abstract}

Keywords Complex Hessian equations $\cdot$ Holder continous solutions $\cdot m$-subharmonic funtions

Mathematics Subject Classifications (2010) 32U05 · 32U40 · 35J99

\section{Introduction}

Let $\Omega$ be an open bounded subset in $\mathbb{C}^{n}$. For $1 \leq m \leq n$, one considers the Dirichlet problem with given $\phi \in C(\partial \Omega)$ and $f \in L^{p}(\Omega), p>n / m$,

$$
\begin{cases}u \in S H_{m} \cap L^{\infty}(\Omega), & \\ \left(d d^{c} u\right)^{m} \wedge \beta^{n-m}=f \beta^{n} & \text { in } \Omega, \\ u=\phi & \text { on } \partial \Omega,\end{cases}
$$

where $S H_{m}(\Omega)$ is the set of $m$-subharmonic functions in $\Omega, \beta=d d^{c}\|z\|^{2}$, and $d=\partial+\bar{\partial}$, $d^{c}=i(\bar{\partial}-\partial)$. In the case $m=1$ (resp. $\left.m=n\right)$ this equation is the Laplace equation for subharmonic functions (resp. the complex Monge-Ampère equation for plurisubharmonic functions).

The complex Monge-Ampère equations have been investigated extensively over last years. We refer the reader to $[3,12,18,22]$, and references therein, for accounts of recent results and more details. We would like to emphasize here that the results on Hölder continuity of solutions of complex Monge-Ampère equations with the right hand side possibly

N. C. Nguyen ( $\square)$

Institute of Mathematics, Jagiellonian University, Łojasiewicza 6, 30-348 Kraków, Poland

e-mail: Nguyen.Ngoc.Cuong@im.uj.edu.pl 
degenerate (see $[8,12,19])$ on compact Kähler manifolds turned out to be very useful in complex dynamic and complex geometry (see e.g. [4, 11]).

On the other hand, the complex Hessian equation is a rather new subject. A major progress has been done recently both for domains in $\mathbb{C}^{n}$ (see $[2,9,20]$ ), and on compact Kähler manifolds, (see [10, 17]). In particular, the Calabi-Yau type theorem for complex Hessian equations on a compact Kähler manifold was proved in [10]. It is expected to have some geometric applications, though not on the scale the complex Monge-Ampère equations have.

The weak solution to complex Hessian equations have been studied in [2, 5, 6, 9, 21]. It has been shown (see $[2,5,9]$ ) that pluripotential theory can be adapted to $m$-subharmonic functions, and it is a suitable tool for studying the weak solution to complex Hessian equations with the right hand side in $L^{p}, p>n / m$. Actually, Dinew and Kołodziej have obtained the continuous solution to the complex Hessian equation for domains in $\mathbb{C}^{n}$ ([9], Theorem 2.10) and for compact Kähler manifolds ([10], Theorem 0.4).

In order to study the Hölder continuous solutions of the complex Hessian equation on a general Kähler manifold it seems that the regularization techniques for $\omega-m$-subharmonic functions ([9], Definition 1.1) will play an important role (see [8, 19]). But in the case $1<m<n$, the problem of the regularization of non smooth $\omega-m$-subharmonic functions for a general Kähler form $\omega$ still needs to be solved. Hence we restrict ourself to the case of domains in $\mathbb{C}^{n}$ with the standard Kähler form $\beta$. Here we wish to study Hölder continuous solutions to Eq. 1.1 in a smoothly bounded, strongly $m$-pseudoconvex domain.

It is also motivated by the result in [14] for $m=n$, where the Eq. 1.1 becomes the complex Monge-Ampère equation, now considered in a strongly pseudoconvex domain. Given $f \in L^{p}(\Omega), p>1, \phi \in C(\partial \Omega)$ one seeks $u$ such that

$$
\begin{cases}u \in P S H \cap L^{\infty}(\Omega), & \\ \left(d d^{c} u\right)^{n}=f d V & \text { in } \Omega, \\ u=\phi & \text { on } \partial \Omega,\end{cases}
$$

where $\operatorname{PSH}(\Omega) \equiv S H_{n}(\Omega), d V:=\beta^{n}$ is the Lebesgue measure. It has been shown that (see [18]) the solution $u$ of Eq. 1.2 is continuous. Later on, in [14] the authors further showed that the solution $u$ belongs to $\operatorname{Lip}_{\alpha}(\bar{\Omega}), \alpha=\alpha(n, p)$, provided some additional assumptions on the boundary data $\phi$ or on the Laplacian mass of $u$.

Our purpose is to prove the counterpart of the above result for complex Hessian equations. More precisely, we want to show that for $1<m<n$ the continuous solution $u$ to Eq. 1.1 obtained in [9] is uniformly Hölder in $\Omega$, under some extra assumptions, by using the potential theory developed in [9] and suitable barrier arguments. The main theorem is as follows.

Theorem 1.1 Let $\Omega$ be a smoothly bounded, strongly m-pseudoconvex domain, $1<m<n$. Let $0 \leq f \in L^{p}(\Omega), p>\frac{n}{m}, \phi \in C^{1,1}(\partial \Omega)$, and let $u$ be the solution of Eq. 1.1.

(a) If $f$ is bounded near the boundary $\partial \Omega$, then $u \in \operatorname{Lip}_{\alpha}(\bar{\Omega})$ for any $0 \leq \alpha<2 \gamma_{1}$;

(b) If $f(z) \leq C|\rho(z)|^{-m v}$ near $\partial \Omega$ for some $C>0,0 \leq v<\frac{1}{2}$, with $\rho$ being the defining function of $\Omega$ as in Eq. 4.12, then $u \in \operatorname{Lip}_{\alpha}(\bar{\Omega})$ for any $0 \leq \alpha<\gamma_{2}$,

where $0<\gamma_{1}, \gamma_{2}<\frac{1}{2}$ are uniform constants defined in Eq. 3.4.

Recently, L.H. Chinh [6] also studied the Hölder continuity of the solution to Eq. 1.1 for $1<m<n$ by the viscosity method. In particular, in connection with our results in the case 
of a domain in $\mathbb{C}^{n}$, he proved Hölder continuity of solutions in the strongly pseudoconvex domains with the right hand side being at least continuous in $\bar{\Omega}$. However, compared to Theorem 1.1, he has put much less regularity on the boundary data $\phi$, namely he took $\phi$ in a Hölder continuous class.

The organization of the paper is as follows, in Section 2 basic notions related to $m$ subharmonic functions are recalled. Section 3 deals with stability estimates. The crucial inequality is Proposition 3.2 due to Dinew and Kołodziej, which fills the gap for the case $1<m<n$ in order to get Theorem 3.6. In Section 4, we first prove a more general statement in Theorem 4.1, and then we verify that under assumptions of Theorem 1.1 one can apply this statement. In particular, Theorem 4.7 will show that any Hölder continuous function on the boundary can be extended to a $m$-sh Hölder continuous function in the whole domain.

\section{$2 m$-subharmonic Functions}

We briefly recall basic notions concerning $m$-subharmonic functions. We refer the reader to $[2,9]$ for a more detailed account. Let $\Omega$ be a bounded open subset in $\mathbb{C}^{n}$. Let $\beta:=d d^{c}\|z\|^{2}$ denote the standard Kähler form in $\mathbb{C}^{n}$, where $d=\partial+\bar{\partial}$ and $d^{c}=i(\bar{\partial}-\partial)$.

\section{1 m-subharmonic Functions}

For $1 \leq m \leq n$ one considers the positive symmetric cone

$$
\Gamma_{m}=\left\{\lambda \in \mathbb{R}^{n}: \sigma_{1}(\lambda)>0, \ldots, \sigma_{m}(\lambda)>0\right\},
$$

where $\sigma_{k}(\lambda):=\sum_{1 \leq i_{1}<\ldots<i_{j} \leq n} \lambda_{i_{1}} \ldots \lambda_{i_{j}}$ are the $k$-th elementary symmetric polynomials of $\lambda$. These symmetric cones are convex (see [13]).

Definition 2.1 Let $u$ be a subharmonic function in $\Omega$.

(a) For smooth case, $u \in C^{2}(\Omega)$ is called $m$-subharmonic (m-sh for short) if the eigenvalue values of the complex Hessian matrix form a vector

$$
\lambda\left[\frac{\partial^{2} u}{\partial z_{j} \partial \bar{z}_{k}}(z)\right] \in \bar{\Gamma}_{m}, \text { equivalently }\left[d d^{c} u(z)\right]^{k} \wedge \beta^{n-k}(z) \geq 0,1 \leq k \leq m, \quad \forall z \in \Omega .
$$

(b) For non-smooth case, $u$ is called $m$-sh if for any collection of $v_{1}, \ldots, v_{m-1} C^{2}$-smooth $m$-sh functions (in the definition (a)) the inequality

$$
d d^{c} u \wedge d d^{c} v_{1} \wedge \ldots \wedge d d^{c} v_{m-1} \wedge \beta^{n-m} \geq 0
$$

holds in the weak sense of currents in $\Omega$.

The set of all $m$-sh functions is denoted by $S H_{m}(\Omega)$.

Following the Bedford and Taylor construction the wedge products of currents given by locally bounded $m$-sh functions are well defined (defined inductively, see also [2]).

Proposition 2.2 Let $u_{1}, \ldots, u_{m}$ be bounded $m$-sh functions then the measure

$$
d d^{c} u_{1} \wedge \ldots \wedge d d^{c} u_{m} \wedge \beta^{n-m}
$$

is nonnegative. 
It can be shown (see [2]) that these nonnegative measures are continuous under monotone or uniform convergence of their potentials.

\section{$2.2 m$-pseudoconvex Domains}

Let $\Omega$ be a bounded domain with $\partial \Omega$ in the class $C^{2}$. Let $\rho \in C^{2}$ in a neighborhood of $\bar{\Omega}$ be a defining function of $\Omega$, i.e. a function such that

$$
\rho<0 \text { on } \Omega, \quad \rho=0 \text { and } d \rho \neq 0 \text { on } \partial \Omega .
$$

Definition 2.3 A $C^{2}$ bounded domain is called strongly $m$-pseudoconvex if there is a defining function $\rho$ and some $\sigma>0$ such that $\left(d d^{c} \rho\right)^{k} \wedge \beta^{n-k} \geq \sigma \beta^{n}$ in $\bar{\Omega}$ for every $1 \leq k \leq m$.

Using the defining function $\rho$ above together with the regularity of the boundary data one can state the following result for subharmonic functions. This proposition seems to be classical. Since we could not find an accurate reference (see [16], Lemma 1.35 and [1], Theorem 6.2 for example), we include its proof, which is based on [1], for the convenience of the reader. This proposition will be used in the proof of Lemma 4.4.

Proposition 2.4 Let $\Omega$ be a smoothly bounded (i.e. strongly 1-pseudoconvex) domain and $\phi \in \operatorname{Lip}_{2 \alpha}(\bar{\Omega}), 0<\alpha \leq \frac{1}{2}$. Then the upper envelope

$$
h(z)=\sup \left\{v(z): v \in S H(\Omega) \cap C(\bar{\Omega}), \quad v_{\mid \partial \Omega} \leq \phi\right\}
$$

belongs to $\operatorname{Lip}_{\tau}(\bar{\Omega})$ for every $0<\tau \leq 2 \alpha<1$ (or for every $0<\tau<1$ when $2 \alpha=1$ ).

Here, and in the whole note, we use the notation:

$$
\operatorname{Lip}_{\alpha}(\bar{\Omega})=\left\{v \in C(\bar{\Omega}):\|v\|_{\alpha}<+\infty\right\},
$$

for $0<\alpha<1$, where the $\alpha$-Hölder norm is given by

$$
\|v\|_{\alpha}:=\sup \{|v(z)|: z \in \bar{\Omega}\}+\sup _{z \neq w}\left\{\frac{|v(z)-v(w)|}{|z-w|^{\alpha}}: z, w \in \bar{\Omega}\right\} .
$$

It is also convenient if we consider in the case $\alpha=0$ the space of continuous functions in $\bar{\Omega}$, and in the case $\alpha=1$ the space of Lipschitz continuous functions with uniform Lipschitz constants in $\bar{\Omega}$.

Proof It is classical fact that $h$ is a harmonic function in $\Omega$ with the boundary value $\phi$, and it belongs to $C(\bar{\Omega})$. In the next step we will construct subharmonic and superharmonic barriers at a given point on the boundary. Let $\rho$ be the strictly subharmonic defining function of $\Omega$.

Lemma 2.5 Suppose that $\|\phi\|_{2 \alpha}=M$, and $0<\tau<1$ such that $\tau \leq 2 \alpha$. Given $\xi \in \partial \Omega$ there is a uniform constant $K=K(\phi, \Omega)>0$ such that the function

$$
a_{\xi}(z)=K|\rho|^{\tau}(z)+M|z-\xi|^{2 \alpha}+\phi(\xi)
$$

is superharmonic in $\Omega \cap W$, where $W$ is a neighborhood of $\partial \Omega$. Moreover, it is equal to $\phi(\xi)$ at $\xi$, and $a_{\xi}(z) \geq \phi(z)$ for every $z \in \partial \Omega$. 
Proof of Lemma 2.5 We have

$$
d d^{c}\left(|\rho|^{\tau}\right)=-\tau|\rho|^{\tau-1} d d^{c} \rho-\tau(1-\tau)|\rho|^{\tau-2} d \rho \wedge d^{c} \rho \text { in } \Omega,
$$

and

$d d^{c}|z-\xi|^{2 \alpha}=\alpha|z-\xi|^{2(\alpha-1)} d d^{c}|z-\xi|^{2}-\alpha(1-\alpha)|z-\xi|^{2(\alpha-2)} d|z-\xi|^{2} \wedge d^{c}|z-\xi|^{2}$.

Hence, we have, in $\Omega$,

$$
d d^{c} a_{\xi}(z) \wedge \beta^{n-1}(z) \leq-K \tau(1-\tau)|\rho|^{\tau-2}|\nabla \rho(z)|^{2} \beta^{n}(z)+M \alpha|z-\xi|^{2(\alpha-1)} \beta^{n}(z) .
$$

Furthermore, there exists $C>0$ such that

$$
|\rho(z)|=|\rho(z)-\rho(\xi)| \leq C|z-\xi| \text { for every } z \in \bar{\Omega} .
$$

Since $\tau-2<0$, it implies that, for $z \in \Omega$,

$$
\begin{aligned}
d d^{c} a_{\xi}(z) \wedge \beta^{n-1}(z) & \leq-c s t . K|z-\xi|^{\tau-2}|\nabla \rho(z)|^{2} \beta^{n}(z)+M \alpha|z-\xi|^{2(\alpha-1)} \beta^{n}(z) \\
& =|z-\xi|^{2(\alpha-1)}\left[M \alpha-\operatorname{cst} . K|z-\xi|^{\tau-2 \alpha}|\nabla \rho(z)|^{2}\right] \beta^{n}(z) \\
& \leq|z-\xi|^{2(\alpha-1)}\left[M \alpha-\operatorname{cst} . K \operatorname{diam}(\Omega)^{\tau-2 \alpha}|\nabla \rho(z)|^{2}\right] \beta^{n}(z),
\end{aligned}
$$

where $c s t=C^{\tau-2} \tau(1-\tau)$, the last inequality follows from the fact that $\tau \leq 2 \alpha$ and $|z-\xi| \leq \operatorname{diam}(\Omega)$. As $\rho$ is a defining function of $\Omega, d \rho \neq 0$ on $\partial \Omega$, one has $|\nabla \rho|>$ $\varepsilon>0$ in a small neighborhood $W$ of $\partial \Omega$. From Eq. 2.3 we get that for $K>0$ big enough, independent of $\xi, a_{\xi}(z)$ is superharmonic in $\Omega \cap W$. The two latter properties follow from the formula for $a_{\xi}(z)$ and $M=\|\phi\|_{2 \alpha}$.

End of Proof of Proposition 2.4 We may extend $a_{\xi}(z)$ to $\Omega$ as follows. Let $U \subset \subset W$ be a neighborhood of $\partial \Omega$, and let $0 \leq \chi \leq 1$ be a smooth cutoff function such that $\chi=1$ in $\bar{U}$, and supp $\chi \subset \subset W$. Since in $\Omega \backslash \bar{U}$ the function $\chi(z) a_{\xi}(z)$ is smooth the function $\tilde{a}_{\xi}(z)=$ $\chi(z) a_{\xi}(z)-K^{\prime} \rho(z)$ is superharmonic in $\Omega$ for $K^{\prime}=K^{\prime}(\Omega, \phi, \rho)>0$ big enough. It is clear that $\tilde{a}_{\xi} \in \operatorname{Lip} p_{\tau}(\bar{\Omega}), \tilde{a}_{\xi}(z) \geq \phi(z)$ on $\partial \Omega$, and $\tilde{a}_{\xi}(\xi)=\phi(\xi)$. Finally, the superharmonic barrier is obtained by setting

$$
a(z):=\inf \left\{\tilde{a}_{\xi}(z): \xi \in \partial \Omega\right\} .
$$

Then $-a(z) \in S H(\Omega) \cap \operatorname{Lip}_{\tau}(\bar{\Omega})$, and $a(z)=\phi(z)$ on $\partial \Omega$. We have constructed a superharmonic function $a(z)$ in $\Omega$, and its boundary value is $\phi$. Similarly, there is a subharmonic function $b \in S H(\Omega) \cap \operatorname{Lip}_{\tau}(\bar{\Omega})$ such that $b \leq h$ in $\Omega, b=\phi$ on $\partial \Omega$. According to the maximum principle, we have

$$
b(z) \leq h(z) \leq a(z)
$$

in $\bar{\Omega}$. Therefore

$$
|h(z)-h(\xi)| \leq K_{1}|z-\xi|^{\tau}, \quad K_{1}=K(a, b),
$$

for every $z \in \bar{\Omega}, \xi \in \partial \Omega$. We will show that Eq. 2.4 holds for any $z, \xi \in \bar{\Omega}$. For any small vector $w \in \mathbb{C}^{n}$, define

$$
V(z, w)= \begin{cases}\max \left\{h(z+w)-K_{1}|w|^{\tau}, h(z)\right\} & z, z+w \in \Omega, \\ h(z) & z \in \bar{\Omega}, z+w \notin \Omega .\end{cases}
$$

Observe that for all $w$, the function $z \rightarrow V(z, w) \in S H(\Omega)$ by Eq. 2.4, and $V(\xi, w)=\phi(\xi)$ on $\partial \Omega$. It follows that for all $z \in \Omega, V(z, w) \leq h(z)$. If $z+w \in \bar{\Omega}$, this yields

$$
h(z+w)-h(z) \leq K_{1}|w|^{\tau} \text {. }
$$


Reversing the roles of $z+w$ and $z$, we obtain

$$
|h(z+w)-h(z)| \leq K_{1}|w|^{\tau} .
$$

Thus, $h \in \operatorname{Lip}_{\tau}(\bar{\Omega})$, and the proposition follows.

\subsection{Comparison Principles}

In next two sections, we will need the following two comparison principles.

Lemma 2.6 (Comparison Principle) Let $\Omega$ be an open bounded subset in $\mathbb{C}^{n}$. For $u, v \in$ $S H_{m} \cap L^{\infty}(\Omega)$ satisfying $\liminf _{\zeta \rightarrow z}(u-v)(\zeta) \geq 0$ for any $z \in \partial \Omega$, we have

$$
\int_{\{u<v\}}\left(d d^{c} v\right)^{m} \wedge \beta^{n-m} \leq \int_{\{u<v\}}\left(d d^{c} u\right)^{m} \wedge \beta^{n-m} .
$$

Consequently, if $\left(d d^{c} u\right)^{m} \wedge \beta^{n-m} \leq\left(d d^{c} v\right)^{m} \wedge \beta^{n-m}$ in $\Omega$, then $v \leq u$ in $\Omega$.

Proof See [21], Theorem 1.14 and Corollary 1.15.

Lemma 2.7 Let $\Omega$ be an open bounded subset in $\mathbb{C}^{n}$. Let $u, v$ be continuous functions on $\bar{\Omega}$ and be $m$-sh functions in $\Omega$. Suppose that $u \leq v$ in $\Omega$ and $u=v$ on $\partial \Omega$. Then,

$$
\begin{gathered}
\int_{\Omega} d d^{c} v \wedge \beta^{n-1} \leq \int_{\Omega} d d^{c} u \wedge \beta^{n-1} \\
\int_{\Omega} d v \wedge d^{c} v \wedge \beta^{n-1} \leq \int_{\Omega} d u \wedge d^{c} u \wedge \beta^{n-1} .
\end{gathered}
$$

Proof The two inequalities are proved in the same way. We will only verify the first one. Set $v_{\varepsilon}=\max \{v-\varepsilon, u\}$ for $\varepsilon>0$. Since $u, v$ are continuous and $u=v$ on $\partial \Omega$, one has $v_{\varepsilon}=u$ in a neighborhood of $\partial \Omega$. Hence

$$
\int_{\Omega} d d^{c} v_{\varepsilon} \wedge \beta^{n-1}=\int_{\Omega} d d^{c} u \wedge \beta^{n-1} .
$$

Moreover, $u \leq v$ in $\Omega$ it implies that $v_{\varepsilon} \nearrow v$ in $\Omega$. Applying the monotone convergence theorem one obtains weak* convergence $d d^{c} v_{\varepsilon} \wedge \beta^{n-1} \rightarrow d d^{c} v \wedge \beta^{n-1}$. This implies

$$
\begin{aligned}
\int_{\Omega} d d^{c} v \wedge \beta^{n-1} & \leq \liminf _{\varepsilon \rightarrow 0} \int_{\Omega} d d^{c} v_{\varepsilon} \wedge \beta^{n-1} \\
& =\int_{\Omega} d d^{c} u \wedge \beta^{n-1} .
\end{aligned}
$$

The lemma follows.

\section{Stability Estimates}

In this section one considers $\Omega$ to be a bounded open set in $\mathbb{C}^{n}$. The main goal is to prove the stability estimate, Theorem 3.6, in the case $1<m<n$. The $m$-capacity, which is the version of the relative capacity of plurisubharmonic functions for $m$-sh functions, will play the analogous role in estimates as in the pluripotential case. For $E$ a Borel set in $\Omega$ we define

$$
\operatorname{cap}_{m}(E, \Omega)=\sup \left\{\int_{E}\left(d d^{c} v\right)^{m} \wedge \beta^{n-m}: v \in S H_{m}(\Omega), 0 \leq v \leq 1\right\} \text {. }
$$


Lemma 3.1 Let $\varphi, \psi \in S H_{m} \cap L^{\infty}(\Omega)$ be such that $\underline{\lim }_{\zeta \rightarrow \partial \Omega}(\varphi-\psi)(\zeta) \geq 0$. Then for all $t, s \geq 0$,

$$
t^{m} \operatorname{cap}_{m}(\{\varphi-\psi<-s-t\}, \Omega) \leq \int_{\{\varphi-\psi<-s\}}\left(d d^{c} \varphi\right)^{m} \wedge \beta^{n-m} .
$$

Proof Take $-1 \leq v \leq 0$ a $m$-sh function in $\Omega$. Since $\{\varphi+s<\psi-t\} \subset\{\varphi+s<\psi+t v\}$, by the comparison principle (Lemma 2.6),

$$
\begin{aligned}
t^{m} \int_{\{\varphi-\psi<-s-t\}}\left(d d^{c} v\right)^{m} \wedge \beta^{n-m} & =\int_{\{\varphi+s<\psi-t\}}\left[d d^{c}(t v)\right]^{m} \wedge \beta^{n-m} \\
& \leq \int_{\{\varphi+s<\psi+t v\}}\left(d d^{c} t v+\psi\right)^{m} \wedge \beta^{n-m} \\
& \leq \int_{\{\varphi+s<\psi+t v\}}\left(d d^{c} \varphi\right)^{m} \wedge \beta^{n-m} \\
& \leq \int_{\{\varphi+s<\psi\}}\left(d d^{c} \varphi\right)^{m} \wedge \beta^{n-m}
\end{aligned}
$$

where the last inequality used $\{\varphi+s<\psi+t v\} \subset\{\varphi+s<\psi\}$.

The following result is an important inequality, due to Dinew and Kołodziej (see [9], Proposition 2.1), between the Euclidean volume and the $m$-capacity of Borel sets.

Proposition 3.2 Let $\Omega$ be a bounded open subset in $\mathbb{C}^{n}$ and $0 \leq \tau<\frac{n}{n-m}$. Then there exists a constant $C=C(\tau)>0$ such that for any Borel subset $E \subset \subset \Omega$,

$$
V(E) \leq C\left[\operatorname{cap}_{m}(E, \Omega)\right]^{\tau},
$$

where $V:=\beta^{n}$ is the volume form.

It helps to obtain the following estimates in the case of $m$-subharmonic functions.

Lemma 3.3 Assume that $0 \leq f \in L^{p}(\Omega), p>\frac{n}{m}$ and $0<\alpha<\frac{p-\frac{n}{m}}{p(n-m)}$. Then there exists a constant $C=C\left(\alpha,\|f\|_{L^{p}(\Omega)}\right)>0$ such that

$$
\int_{E} f d V \leq C\left[\operatorname{cap}_{m}(E, \Omega)\right]^{1+\alpha m}
$$

for every $E \subset \subset \Omega$.

Proof Applying Hölder's inequality and then using Eq. 3.1 with $\tau=(1+\alpha m) \frac{p}{p-1}<\frac{n}{n-m}$ we have

$$
\begin{aligned}
\int_{E} f d V & \leq\|f\|_{L^{p}(\Omega)}[V(E)]^{\frac{1}{q}} \leq C(\tau)\|f\|_{L^{p}(\Omega)}\left[\operatorname{cap}_{m}(E, \Omega)\right]^{\frac{\tau}{q}} \\
& \leq C\left(\alpha,\|f\|_{L^{p}(\Omega)}\left[\operatorname{cap}_{m}(E, \Omega)\right]^{1+\alpha m}\right.
\end{aligned}
$$

where $\frac{1}{p}+\frac{1}{q}=1$. Hence, the lemma follows.

The following lemma was proved in [12]. 
Lemma 3.4 Let $g: \mathbb{R}^{+} \rightarrow \mathbb{R}^{+}$be a decreasing right continuous function. Assume there exists $\alpha>0$ and $B>0$ such that

$$
\operatorname{tg}(s+t) \leq B[g(s)]^{1+\alpha} \text { for every } s, t \geq 0 .
$$

Then $g(s)=0$ for all $s \geq s_{\infty}$, where $s_{\infty}:=\frac{2 B[g(0)]^{\alpha}}{1-2^{-\alpha}}$.

Proof See Lemma 2.4 in [12]. The additional point is that the condition (3.2) holds for every $s, t \geq 0$ while in [12] the assumptions are for every $s \geq 0$ and for every $0 \leq t \leq 1$. Therefore, we may compute $s_{\infty}$ as in the statement.

By combining Lemma 3.1, Lemma 3.3 and Lemma 3.4, we get

Proposition 3.5 Let $\varphi, \psi \in S H_{m} \cap L^{\infty}(\Omega)$ be such that $\underline{\lim }_{\zeta \rightarrow \partial \Omega}(\varphi-\psi)(\zeta) \geq 0$, and $0 \leq f \in L^{p}(\Omega), p>\frac{n}{m}$. Suppose that $\left(d d^{c} \varphi\right)^{m} \wedge \beta^{n-m}=f \beta^{n}$ in $\Omega$ and $0<\alpha<\frac{p-\frac{n}{m}}{p(n-m)}$. Then there exists a constant $A=A\left(\alpha,\|f\|_{L^{p}(\Omega)}\right)$ such that for all $\varepsilon>0$,

$$
\sup _{\Omega}(\psi-\varphi) \leq \varepsilon+A\left[\operatorname{cap}_{m}(\{\varphi-\psi<-\varepsilon\}, \Omega)\right]^{\alpha} .
$$

Proof Put $g(s):=\left[\operatorname{cap}_{m}(\{\varphi-\psi<-\varepsilon-s\}, \Omega)\right]^{\frac{1}{m}}$. Applying in turn Lemma 3.1 and Lemma 3.3, we obtain

$$
\begin{aligned}
t^{m} \operatorname{cap}_{m}(\{\varphi-\psi<-\varepsilon-s-t\}, \Omega) & \leq \int_{\{\varphi-\psi<-\varepsilon-s\}}\left(d d^{c} \varphi\right)^{m} \wedge \beta^{n-m} \\
& =\int_{\{\varphi-\psi<-\varepsilon-s\}} f \beta^{n} \\
& \leq C\left(\alpha,\|f\|_{L^{p}(\Omega)}\right)\left[\operatorname{cap}_{m}(\{\varphi-\psi<-\varepsilon-s\}, \Omega)\right]^{1+\alpha m} .
\end{aligned}
$$

Now, taking the m-th root of two sides one gets that

$$
\operatorname{tg}(s+t) \leq B[g(s)]^{1+\alpha m} \quad \text { with } \quad B=C^{\frac{1}{m}}\left(\alpha,\|f\|_{L^{p}(\Omega)}\right) .
$$

From Eq. 3.3 we see that $g(s)$ satisfies assumptions of Lemma 3.4. It tells us that $\left[g\left(s_{\infty}\right)\right]^{m}=\operatorname{cap}_{m}\left(\left\{\varphi-\psi<-\varepsilon-s_{\infty}\right\}, \Omega\right)=0$, which means $\psi-\varphi \leq \varepsilon+s_{\infty}$ almost everywhere. Finally, by inserting into formula $s_{\infty}=\frac{2 B[g(0)]^{\alpha m}}{1-2^{-\alpha m}}$ we obtain

$$
\sup _{\Omega}(\psi-\varphi) \leq \varepsilon+A\left[\operatorname{cap}_{m}(\{\varphi-\psi<-\varepsilon\}, \Omega)\right]^{\alpha},
$$

where $A=\frac{2 B}{1-2^{-\alpha m}}$.

We are now in the position to prove the main stability estimate which is similar to Theorem 1.1 in [14] for $m=n$ (see also [9], Theorem 2.5 in the case $1<m<n$ ). In order to simplify the notation, from now on when $p>\frac{n}{m}, 1<m<n, \frac{1}{p}+\frac{1}{q}=1$ we set

$$
\gamma_{r}:=\frac{r}{r+m q+\frac{p q(n-m)}{p-\frac{n}{m}}}, \quad \text { for } r \geq 1
$$


Theorem 3.6 (Stability Estimate) Let $\varphi, \psi \in S H_{m} \cap L^{\infty}(\Omega)$ be such that $\underline{\lim }_{\zeta \rightarrow \partial \Omega}(\varphi-\psi)(\zeta) \geq 0$, and $0 \leq f \in L^{p}(\Omega), p>\frac{n}{m}$. Suppose that $\left(d d^{c} \varphi\right)^{m} \wedge \beta^{n-m}=$ $\overrightarrow{f \beta^{n}}$ in $\Omega$. Fix $r \geq 1$ and $0<\gamma<\gamma_{r}$. Then there exists a constant $C=C\left(\gamma,\|f\|_{L^{p}(\Omega)}\right)>0$ such that

$$
\sup _{\Omega}(\psi-\varphi) \leq C\left[\left\|(\psi-\varphi)_{+}\right\|_{L^{r}(\Omega)}\right]^{\gamma},
$$

where $(\psi-\varphi)_{+}=\max \{\psi-\varphi, 0\}$.

Proof We follow the lines of the proof of Theorem 1.1 in [14]. Applying Lemma 3.1 with $s=t=\varepsilon>0$ and then using Hölder's inequality, we get

$$
\begin{aligned}
\operatorname{cap}_{m}(\{\varphi-\psi<-2 \varepsilon\}, \Omega) & \leq \varepsilon^{-m} \int_{\{\varphi-\psi<-\varepsilon\}} f d V \\
& \leq \varepsilon^{-m-\frac{r}{q}} \int_{\Omega}(\psi-\varphi)_{+}^{\frac{r}{q}} f d V \\
& \leq \varepsilon^{-m-\frac{r}{q}}\|f\|_{L^{p}(\Omega)}\left\|(\psi-\varphi)_{+}\right\|_{L^{r}(\Omega)}^{\frac{r}{q}} .
\end{aligned}
$$

Fix $0<\alpha<\frac{p-\frac{n}{m}}{p(n-m)}$ to be chosen later. Applying Proposition 3.5 we have

$$
\sup _{\Omega}(\psi-\varphi) \leq 2 \varepsilon+A \varepsilon^{-\alpha\left(m+\frac{r}{q}\right)}\|f\|_{L^{p}(\Omega)}^{\alpha}\left\|(\psi-\varphi)_{+}\right\|_{L^{r}(\Omega)}^{\frac{\alpha r}{q}},
$$

where $A=A\left(\alpha,\|f\|_{L^{p}(\Omega)}\right)$. Now, we choose $\varepsilon=\left\|\left(\psi-\varphi_{+}\right)\right\|_{L^{r}(\Omega)}^{\gamma}$ and $\alpha=\frac{\gamma q}{r-\gamma(m q+r)}$ which is well defined, the condition $0<\gamma<\gamma_{r}$ being equivalent to $0<\alpha<\frac{p-\frac{n}{m}}{p(n-m)}$. Then the inequality (3.5) becomes

$$
\sup _{\Omega}(\psi-\varphi) \leq A\|f\|_{L^{p}(\Omega)}^{\alpha}\left\|(\psi-\varphi)_{+}\right\|_{L^{r}(\Omega)}^{\gamma}
$$

Thus, the theorem is proved.

\section{Hölder Continuity of the Solution}

Let $\Omega$ be a smooth bounded, strongly $m$-pseudoconvex domain in $\mathbb{C}^{n}, 1<m<n$. We consider the Dirichlet problem for the complex Hessian equation in the class of $m$-sh functions.

$$
\begin{cases}\left(d d^{c} u\right)^{m} \wedge \beta^{n-m}=f \beta^{n} & \text { in } \Omega \\ u=\phi & \text { on } \partial \Omega\end{cases}
$$

From the recent result of Dinew and Kołodziej (see [9], Theorem 2.10) we know that $u \in S H_{m}(\Omega) \cap C(\bar{\Omega})$ when $f \in L^{p}(\Omega), p>n / m$ and $\phi \in C(\partial \Omega)$. After establishing the stability estimates in Section 3, we may use the scheme of the proof in [14] in order to obtain further the Hölder continuity of the solution $u$ to Eq. 4.1 under some additional assumptions. 
Theorem 4.1 Let $0 \leq f \in L^{p}(\Omega), p>\frac{n}{m}$, and $\phi \in C(\partial \Omega)$. Let $u$ be the continuous solution to Eq. 4.1. Suppose that there exists $b \in \operatorname{Lip}_{v}(\bar{\Omega}), 0<v<1$, such that $b \leq u$ in $\Omega, b=u$ on $\partial \Omega$.

(a) If $\nabla u$ belongs to $L^{2}(\Omega)$, then $u \in \operatorname{Lip}_{\alpha}(\bar{\Omega})$ for any $0 \leq \alpha<\min \left\{v, \gamma_{2}\right\}$.

(b) If the total mass of $\Delta u$ is finite, then $u \in \operatorname{Lip}(\bar{\Omega})$ for any $0 \leq \alpha<\min \left\{v, 2 \gamma_{1}\right\}$.

Where $\gamma_{1}, \gamma_{2}$ are defined in Eq. 3.4.

It is not too difficult to see that when the total mass of $\Delta u$ is finite, $\nabla u \in L^{2}(\Omega)$. However, the Hölder exponent in Theorem 4.1-(b) is better than the one in Theorem 4.1-(a), namely $\gamma_{2}<2 \gamma_{1}$. If we put some extra assumptions on the growth of the density $f$ near the boundary and on the boundary data $\phi$, then we may verify the assumptions of Theorem 4.1, which is the content of the main theorem (Theorem 1.1).

Proof of Theorem 4.1 For a fixed $\delta>0$, we set

$$
\begin{gathered}
\Omega_{\delta}:=\{z \in \Omega: \operatorname{dist}(z, \partial \Omega)>\delta\} ; \\
u_{\delta}(z):=\sup _{\|\zeta\| \leq \delta} u(z+\zeta), \quad z \in \Omega_{\delta} ; \\
\hat{u}_{\delta}(z):=\frac{1}{v_{2 n} \delta^{2 n}} \int_{|\zeta-z| \leq \delta} u(\zeta) d V_{2 n}(\zeta), \quad z \in \Omega_{\delta},
\end{gathered}
$$

where $v_{2 n}$ is the volume of the unit ball in $\mathbb{C}^{n}$. The following lemma shows that the Hölder norm (see Eq. 2.2) of $u$ in $\bar{\Omega}$ can be computed by using either Eq. 4.2 or Eq. 4.3.

Lemma 4.2 Given $0<\alpha<1$, the following two conditions are equivalent.

(i) There exists $\delta_{1}, A_{1}>0$ such that for any $0<\delta \leq \delta_{1}$,

$$
u_{\delta}-u \leq A_{1} \delta^{\alpha} \quad \text { on } \quad \Omega_{\delta} .
$$

(ii) There exists $\delta_{2}, A_{2}>0$ such that for any $0<\delta \leq \delta_{2}$,

$$
\hat{u}_{\delta}-u \leq A_{2} \delta^{\alpha} \text { on } \Omega_{\delta} .
$$

Proof See Lemma 4.2 in [14] where its proof used only the subharmonicity.

The assumption $\nabla u \in L^{2}(\Omega)$ (resp. $\left.\|\Delta u\|(\Omega)<+\infty\right)$ will enable us to control the growth of $\left\|u_{\delta}-u\right\|_{L^{2}\left(\Omega_{\delta}\right)}$ (resp. $\left.\left\|\hat{u}_{\delta}-u\right\|_{L^{1}\left(\Omega_{\delta}\right)}\right)$.

Lemma 4.3 For $\delta>0$ small enough, we have inequalities

$$
\begin{gathered}
\int_{\Omega_{\delta}}\left|u_{\delta}(z)-u(z)\right|^{2} d V_{2 n}(z) \leq c_{n}\|\nabla u\|_{L^{2}(\Omega)}^{2} \delta^{2}, \\
\int_{\Omega_{\delta}}\left[\hat{u}_{\delta}(z)-u(z)\right] d V_{2 n}(z) \leq c_{n}\|\Delta u\|_{\Omega} \delta^{2},
\end{gathered}
$$

where $c_{n}>0$ depends only on $n$.

Proof See Lemma 4.3 and the last part in the proof of Theorem 3.1 in [14]. There, only the subharmonicity was needed. 
In view of Lemma 4.2 and Lemma 4.3 we wish to apply the stability estimate, Theorem 3.6, to $\varphi:=u$ and $\psi:=u_{\delta}$. This will give us the Hölder norm estimate in $\bar{\Omega}$ of the solution $u$, in terms of $L^{2}$ norm of its gradient or its Laplacian mass in $\Omega$, using Eq. 4.4 or 4.5. The remaining thing that we need is extending $u_{\delta}$ to $\Omega$ (since it is only defined on $\Omega_{\delta}$ ), in such a way that after the extension the Hölder norm of $u$ is still under control. It will be done with the help of the barrier function $b \in \operatorname{Lip}_{v}(\bar{\Omega}), 0<v<1$.

Lemma 4.4 Under the assumptions of Theorem 4.1, there exists a constant $c_{0}=$ $c_{0}(b, \Omega)>0$ and $\delta_{0}$ small enough such that for any $0<\delta<\delta_{0}$

$$
u_{\delta}(z) \leq u(z)+c_{0} \delta^{v} \text { for every } z \in \partial \Omega_{\delta},
$$

where $v$ is the Hölder exponent of $b$. Consequently, the function

$$
\tilde{u}_{\delta}= \begin{cases}\max \left\{u_{\delta}, u+c_{0} \delta^{\nu}\right\} & \text { in } \Omega_{\delta}, \\ u+c_{0} \delta^{\nu} & \text { in } \Omega \backslash \Omega_{\delta}\end{cases}
$$

is a $m$-subharmonic function in $\Omega$, and it is continuous in $\bar{\Omega}$.

Proof Let $h$ be the harmonic extension to $\Omega$ with $b$ as the boundary value on $\partial \Omega$. By Proposition $2.4 h \in \operatorname{Lip}_{v}(\bar{\Omega})$. It is clear that $b \leq u \leq h$ in $\Omega$. Fix a point $z \in \partial \Omega_{\delta}$, there is $\zeta \in \mathbb{C}^{n}$ with $\|\zeta\|=\delta$ such that $z+\zeta \in \bar{\Omega}$ and $u_{\delta}(z)=u(z+\zeta)$. This yields

$$
\begin{aligned}
u_{\delta}(z)-u(z) & =u(z+\zeta)-u(z) \\
& \leq h(z+\zeta)-u(z) \\
& \leq h(z+\zeta)-b(z) .
\end{aligned}
$$

Now, choose $\zeta_{0} \in \mathbb{C}^{n},\left\|\zeta_{0}\right\|=\delta$, such that $z+\zeta_{0} \in \partial \Omega$. It implies that $h\left(z+\zeta_{0}\right)=b\left(z+\zeta_{0}\right)$ as $b=u=h$ on $\partial \Omega$. Then,

$$
\begin{aligned}
h(z+\zeta)-b(z) & =[h(z+\zeta)-h(z)]+[h(z)-b(z)] \\
& \leq\|h\|_{\nu} \delta^{\nu}+\left[h(z)-h\left(z+\zeta_{0}\right)\right]+\left[b\left(z+\zeta_{0}\right)-b(z)\right] \\
& \leq c_{0} \delta^{v} \text { with } c_{0}=2\|h\|_{\nu}+\|b\|_{\nu} .
\end{aligned}
$$

From Eqs. 4.8 and 4.9 we obtain Eq. 4.6. Properties of $\tilde{u}_{\delta}$ in Eq. 4.7 follow from the standard gluing procedure.

Proof of (a) in Theorem 4.1 Given $0<\gamma<\gamma_{2}$. Applying Theorem 3.6 with $\varphi:=u+c_{0} \delta^{\nu}$, $\psi:=\tilde{u}_{\delta}$ and $r=2$ we get

$$
\sup _{\Omega}\left[\tilde{u}_{\delta}-\left(u+c_{0} \delta^{\nu}\right)\right] \leq C\left\|\left(\tilde{u}_{\delta}-u-c_{0} \delta^{\nu}\right)_{+}\right\|_{L^{2}(\Omega)}^{\gamma} .
$$

Since $\tilde{u}_{\delta}=u+c_{0} \delta^{v}$ in $\Omega \backslash \Omega_{\delta}$, it implies that

$$
\sup _{\Omega_{\delta}}\left(u_{\delta}-u-c_{0} \delta^{\nu}\right) \leq C\left\|\left(u_{\delta}-u-c_{0} \delta^{\nu}\right)+\right\|_{L^{2}\left(\Omega_{\delta}\right)}^{\gamma} .
$$

As $\left(u_{\delta}-u-c_{0} \delta^{v}\right)_{+} \leq u_{\delta}-u$ and using Eq. 4.4, we have

$$
\sup _{\Omega_{\delta}}\left(u_{\delta}-u\right) \leq c_{0} \delta^{\nu}+C\left\|u_{\delta}-u\right\|_{L^{2}\left(\Omega_{\delta}\right)}^{\gamma} \leq c_{0} \delta^{\nu}+C \cdot c_{n}^{\frac{\gamma}{2}}\|\nabla u\|_{L^{2}(\Omega)}^{\gamma} \delta^{\gamma} .
$$


Hence,

$$
\sup _{\Omega_{\delta}}\left(u_{\delta}-u\right) \leq c_{1} \delta^{\min \{v, \gamma\}} \text { for } \delta \text { small enough, }
$$

where $c_{1}=c_{0}+C \cdot c_{n}^{\frac{\gamma}{2}}\|\nabla u\|_{L^{2}(\Omega)}^{\gamma}$. This finishes the first part of Theorem 4.1.

Proof of (b) in Theorem 4.1 Given $0<\gamma<\gamma_{1}$. The formula (4.6) implies $\hat{u}_{\delta} \leq u_{\delta} \leq$ $u+c_{0} \delta^{\nu}$ on $\partial \Omega_{\delta}$. Therefore, the function

$$
u_{\delta}^{\prime}= \begin{cases}\max \left\{\hat{u}_{\delta}, u+c_{0} \delta^{\nu}\right\} & \text { in } \Omega_{\delta}, \\ u+c_{0} \delta^{v} & \text { in } \Omega \backslash \Omega_{\delta}\end{cases}
$$

is $m$-subharmonic in $\Omega$, and it is continuous in $\bar{\Omega}$. Applying again Theorem 3.6 with $\varphi:=$ $u+c_{0} \delta^{\nu}, \psi:=u_{\delta}^{\prime}$ and $r=1$ we get

$$
\sup _{\Omega}\left[u_{\delta}^{\prime}-\left(u+c_{0} \delta^{\nu}\right)\right] \leq C\left\|\left(u_{\delta}^{\prime}-u-c_{0} \delta^{\nu}\right)_{+}\right\|_{L^{1}(\Omega)}^{\gamma} .
$$

Since $u_{\delta}^{\prime}=u+c_{0} \delta^{v}$ in $\Omega \backslash \Omega_{\delta}$, it follows that

$$
\sup _{\Omega_{\delta}}\left(\hat{u}_{\delta}-u-c_{0} \delta^{v}\right) \leq C\left\|\left(\hat{u}_{\delta}-u-c_{0} \delta^{\nu}\right)_{+}\right\|_{L^{1}\left(\Omega_{\delta}\right)}^{\gamma} .
$$

Since $\left(\hat{u}_{\delta}-u-c_{0} \delta^{\nu}\right)_{+} \leq \hat{u}_{\delta}-u$ and using Eq. 4.5, we get

$$
\sup _{\Omega_{\delta}}\left(\hat{u}_{\delta}-u\right) \leq c_{0} \delta^{\nu}+C\left\|\hat{u}_{\delta}-u\right\|_{L^{1}\left(\Omega_{\delta}\right)}^{\gamma} \leq c_{0} \delta^{\nu}+C \cdot c_{n}^{\gamma}\|\Delta u\|_{\Omega}^{\gamma} \delta^{2 \gamma} .
$$

Hence,

$$
\sup _{\Omega_{\delta}}\left(\hat{u}_{\delta}-u\right) \leq c_{2} \delta^{\min \{v, 2 \gamma\}} \text { for } \delta \text { small enough, }
$$

where $c_{2}=c_{0}+C \cdot c_{n}^{\gamma}\|\Delta u\|_{\Omega}^{\gamma}$. Applying Lemma 4.2 one obtains

$$
\sup _{\Omega_{\delta}}\left(u_{\delta}-u\right) \leq c_{3} \delta^{\min \{v, 2 \gamma\}} \text { for } \delta \text { small enough, }
$$

for some uniform constant $c_{3}>0$. This proves the second part.

Thus, we have finished the proof of Theorem 4.1.

We proceed to prove Theorem 1.1. We fix a defining function $\rho$ of $\Omega$, setting

$$
\begin{aligned}
& \Omega=\{\rho<0\}, \quad \rho \in C^{2}(\bar{\Omega}), \\
& \left(d d^{c} \rho\right)^{k} \wedge \beta^{n-k} \geq \sigma \beta^{n} \text { on } \bar{\Omega}, \\
& 1 \leq k \leq m, \quad \sigma>0 .
\end{aligned}
$$

We first prove the following two lemmas. The first one (cf. Proposition 2.4) was proved in [7] (see also [1]) for $m=n$.

Lemma 4.5 If $\phi \in C^{1,1}(\partial \Omega)$, then the upper envelope

$$
h(z)=\sup \left\{v(z): v \in S H_{m}(\Omega) \cap C(\bar{\Omega}), v \leq \phi \text { on } \partial \Omega\right\}
$$

is a $m$-subharmonic function in $\Omega$ and is Lipschitz continuous in $\bar{\Omega}$. It satisfies $h=\phi$ on $\partial \Omega$. Moreover,

$$
\int_{\Omega} d d^{c} h \wedge \beta^{n-1}<+\infty
$$


Proof It is clear that $h \in S H_{m}(\Omega) \cap C(\bar{\Omega})$, and $h=\phi$ on $\partial \Omega$ because it coincides with the unique continuous solution to Eq. 4.1, where $f \equiv 0$. We consider $\rho$ defined in Eq. 4.12. There is an extension $\hat{\phi}$ of $\phi$ to a neighborhood $U \supset \bar{\Omega}$ such that $\|\hat{\phi}\|_{C^{1,1}(\bar{U})} \leq$ $C\|\phi\|_{C^{1,1}(\partial \Omega)}$, where $C=C(\Omega, U)>0$ (see [15], Lemma 6.38). Hence, for $A>0$ big enough, $A \rho \pm \hat{\phi}$ belongs to $S H_{m}(U)$. Moreover, we can take $C$ so big that

$$
\|A \rho \pm \hat{\phi}\|_{C^{1,1}(\bar{U})} \leq C\left(1+\|\phi\|_{C^{1,1}(\partial \Omega)}\right) .
$$

The definition of $h$ implies

$$
A \rho+\hat{\phi} \leq h \leq \hat{\phi}-A \rho \text { in } \Omega,
$$

where the second inequality follows from the maximum principle for the subharmonic function $h+A \rho-\hat{\phi}$ in $\Omega$. We now extend $h$ to $\bar{U}$ by putting

$$
\hat{h}(z)= \begin{cases}h(z) & \text { for } z \in \Omega, \\ A \rho(z)+\hat{\phi}(z) & \text { for } z \in \bar{U} \backslash \Omega .\end{cases}
$$

According to Eqs. 4.15 and 4.16, $\hat{h} \leq \max \{\hat{\phi}-A \rho, \hat{\phi}+A \rho\}$ in $\bar{U}$. For $\xi \in \partial \Omega,|w|$ so small that $\xi+w \in U$, we have

$$
\begin{aligned}
\hat{h}(\xi+w) & \leq \phi(\xi)+\max \left\{\|\hat{\phi}-A \rho\|_{C^{1}(\bar{U})},\|\hat{\phi}+A \rho\|_{C^{1}(\bar{U})}\right\}|w| \\
& \leq \phi(\xi)+C^{\prime}|w|,
\end{aligned}
$$

where the last inequality follows from Eq. 4.14 with $C^{\prime}=C\left(1+\|\phi\|_{C^{1,1}(\partial \Omega)}\right)$. It implies that

$$
\hat{h}(\xi+w)-C^{\prime}|w| \leq \phi(\xi) \text { for every } \xi \in \partial \Omega .
$$

Hence, from the definition of $h, \hat{h}(z+w)-C^{\prime}|w| \leq h(z)$ in $\bar{\Omega}$. By changing $w$ into $-w$, we get, for $|w|$ so small that $z+w \in \bar{\Omega}$,

$$
|h(z+w)-h(z)| \leq C^{\prime}|w| \text { for } z \in \bar{\Omega},
$$

since $\hat{h}(z)=h(z)$ in $\bar{\Omega}$. Thus $\|h\|_{C^{1}(\bar{\Omega})} \leq C^{\prime}=C\left(1+\|\phi\|_{C^{1,1}(\partial \Omega)}\right)$, in particular $h$ is Lipschitz continuous in $\bar{\Omega}$. Since $h=\phi$ on $\partial \Omega$ and $A \rho+\hat{\phi} \leq h$ it implies

$$
\int_{\Omega} d d^{c} h \wedge \beta^{n-1} \leq \int_{\Omega} d d^{c}(A \rho+\hat{\phi}) \wedge \beta^{n-1}<+\infty
$$

by Lemma 2.7. This verifies (4.13). The proof is finished.

Lemma 4.6 For $0 \leq v<\frac{1}{2}$, the function $\rho_{v}=-|\rho|^{1-v}$, $\rho$ as in Eq. 4.12, belongs to $S H_{m}(\Omega) \cap$ Lip $_{1-v}(\bar{\Omega})$ and satisfies

$$
\int_{\Omega} d \rho_{\nu} \wedge d^{c} \rho_{\nu} \wedge \beta^{n-1}<+\infty
$$

Proof It follows from formulas

$$
d d^{c}\left[-(-\rho)^{1-v}\right]=(1-v)|\rho|^{-v} d d^{c} \rho+v(1-v)|\rho|^{-1-v} d \rho \wedge d^{c} \rho,
$$

and

$$
d \rho_{\nu} \wedge d^{c} \rho_{\nu} \wedge \beta^{n-1}=(1-v)^{2}|\rho|^{-2 v} d \rho \wedge d^{c} \rho \wedge \beta^{n-1} .
$$

Since $-2 v>-1$, the integral (4.17) converges. 
We are now in the position to prove Theorem 1.1. The proof will make use of the envelope $h$ in Lemma 4.5, $\rho_{v}$ in Lemma 4.6 and $\rho$ from Eq. 4.12. In what follows, we will use these functions without mentioning them anymore.

Proof of $(a)$ in Theorem 1.1 Since $f$ is bounded near the boundary there is a compact set $F \subset \subset \Omega$ and $M>0$ such that $0 \leq f \leq M$ in $\Omega \backslash F$. We may choose $A>0$ big enough such that $A \rho+h \leq u$ in a neighborhood of $F$, as $\rho<-\varepsilon$ in $F$ for some $\varepsilon>0$, and

$$
\left[d d^{c}(A \rho+h)\right]^{m} \wedge \beta^{n-m} \geq\left(d d^{c} A \rho\right)^{m} \wedge \beta^{n-m} \geq f \beta^{n} \text { in } \Omega \backslash F .
$$

Therefore, $A \rho+h \leq u$ in $\Omega \backslash F$ by the comparison principle (Lemma 2.6). Therefore,

$$
b:=A \rho+h \leq u \text { in } \Omega \text { and } b \text { is Lipschitz continuous in } \bar{\Omega} \text {. }
$$

Using Lemma 2.7, Eq. 4.13 and the fact that $\rho$ is $C^{2}$ smooth in a neighborhood of $\bar{\Omega}$, we get

$$
\int_{\Omega} d d^{c} u \wedge \beta^{n-1} \leq \int_{\Omega} d d^{c} b \wedge \beta^{n-1}<+\infty
$$

According to Eqs. 4.20 and 4.21, the assumptions of Theorem 4.1-(b) are satisfied. The first part of Theorem 1.1 follows.

Proof of $(b)$ in Theorem 1.1 From the assumption of $f$ near the boundary, there is a compact subset $F \subset \subset \Omega$ such that $f(z) \leq C|\rho|^{-m v}$ in $\Omega \backslash F$. Using Eqs. 4.18 and 4.12, it follows that

$$
\begin{aligned}
\left(d d^{c} \rho_{\nu}\right)^{m} \wedge \beta^{n-m} & \geq(1-v)^{m}(-\rho)^{-m v} \sigma \beta^{n} \\
& \geq \frac{\sigma(1-v)^{m}}{C} f \beta^{n} \quad \text { in } \quad \Omega \backslash F .
\end{aligned}
$$

Therefore, we may choose $A>0$ so big that

$$
b_{v}:=A \rho_{v}+h \leq u \text { in a neighborhood of } F,
$$

and

$$
\left(d d^{c} b_{v}\right)^{m} \wedge \beta^{n-m} \geq\left(d d^{c} A \rho_{\nu}\right)^{m} \wedge \beta^{n-m} \geq f \beta^{n} \quad \text { in } \quad \Omega \backslash F .
$$

Hence, by the comparison principle (Lemma 2.6), we get

$$
b_{v} \leq u \text { in } \Omega \backslash F \text {. }
$$

So,

$$
b_{v} \leq u \text { in } \Omega \text { and } b_{v} \in \operatorname{Lip}_{1-v}(\bar{\Omega}) .
$$

Moreover, Lemma 2.7 and Eq. 4.17 imply

$$
\int_{\Omega} d u \wedge d^{c} u \wedge \beta^{n-1} \leq \int_{\Omega} d b_{v} \wedge d^{c} b_{v} \wedge \beta^{n-1}<+\infty .
$$

According to Eqs. 4.22 and 4.23, the assumptions of Theorem 4.1-(a) are satisfied. Note that $\gamma_{2}<\frac{1}{2}<1-v$. Thus, the second part in Theorem 1.1 follows.

In the last part we consider the homogeneous case of the Eq. 4.1, i.e. the right hand side $f \equiv 0$, when the boundary data is only Hölder continuous. Similarly to the classical case $m=1$, Proposition 2.4 and the case $m=n$, Theorem 6.2 in [1], it says that any Hölder continuous function on the boundary $\partial \Omega$ can be extended to a Hölder continuous $m$ subharmonic function in $\Omega$. For $m=n$, it has been shown in [1] that the Hölder exponent is sharp. 
Theorem 4.7 Let $\Omega$ be a smoothly bounded strongly m-pseudoconvex domain and let $\phi$ belong to $\operatorname{Lip}_{2 \alpha}(\bar{\Omega}), 0<\alpha \leq \frac{1}{2}$. Then the upper envelope

$$
h_{m}(z)=\sup \left\{v(z): v \in S H_{m}(\Omega) \cap C(\bar{\Omega}), \quad v_{\left.\right|_{\partial \Omega}} \leq \phi\right\}
$$

is $m$-subharmonic in $\Omega$, and it belongs to Lip $p_{\alpha}(\bar{\Omega})$.

Proof It is enough to verify the Hölder continuity of $h_{m}(z)$ since $m$-subharmonicity has been shown in [2]. As in the proof of Proposition 2.4, one needs the following $m$-subharmonic barrier at any given point on the boundary. Let $\rho$ be defined in Eq. 4.12.

Lemma 4.8 Suppose that $\|\phi\|_{2 \alpha}=$ M. There is a uniform constant $K=K(\Omega, \rho)>0$ such that for any $\xi \in \partial \Omega$ the function

$$
b_{\xi}(z)=-M\left(|z-\xi|^{2}-K \rho\right)^{\alpha}+\phi(\xi)
$$

is $m$-subharmonic in $\Omega$ and belongs to Lip $(\bar{\Omega})$. Moreover, it is equal to $\phi(\xi)$ at $\xi$, and $b_{\xi}(z) \leq \phi(z)$ for every $z \in \partial \Omega$.

Proof of Lemma 4.8 We have

$$
\begin{aligned}
d d^{c}\left(|z-\xi|^{2}-K \rho\right)^{\alpha}= & \alpha\left(|z-\xi|^{2}-K \rho(z)\right)^{\alpha-1} d d^{c}\left(|z-\xi|^{2}-K \rho\right) \\
& -\alpha(1-\alpha)\left(|z-\xi|^{2}-K \rho(z)\right)^{\alpha-2} d\left(|z-\xi|^{2}-K \rho\right) \\
\wedge & d^{c}\left(|z-\xi|^{2}-K \rho\right) .
\end{aligned}
$$

Hence, in $\Omega$

$$
d d^{c} b_{\xi}(z)=M \alpha\left(|z-\xi|^{2}-K \rho(z)\right)^{\alpha-1} d d^{c}\left(K \rho(z)-|z|^{2}\right)+\Lambda(z, \xi),
$$

where

$$
\Lambda(z, \xi)=M \alpha(1-\alpha)\left(|z-\xi|^{2}-K \rho(z)\right)^{\alpha-2} d\left(|z-\xi|^{2}-K \rho\right) \wedge d^{c}\left(|z-\xi|^{2}-K \rho\right)
$$

is a positive $(1,1)$ form for any $z \in \Omega$. Thus,

$$
d d^{c} b_{\xi}(z)=\Theta(z, \xi)+\Lambda(z, \xi),
$$

where $\Theta(z, \xi)=M \alpha\left(|z-\xi|^{2}-K \rho(z)\right)^{\alpha-1} d d^{c}\left[K \rho(z)-|z|^{2}\right]$. If we choose $K=$ $K(\Omega, \rho)>0$ big enough, independent of $\xi$, then $K \rho(z)-|z|^{2}$ is a strictly $m$-sh function in a neighborhood of $\bar{\Omega}$. It implies that $\Theta(z, \xi) \in \Gamma_{m}$, i.e. the eigenvalues of the matrix of coefficients $\Theta(z, \xi)$ form a vector in $\Gamma_{m}$, for every $z \in \Omega$. Hence, for $1 \leq k \leq m$

$$
\left[d d^{c} b_{\xi}(z)\right]^{k} \wedge \beta^{n-k}(z)=[\Theta(z, \xi)+\Lambda(z, \xi)]^{k} \wedge \beta^{n-k}(z)>0
$$

for every $z \in \Omega$. Therefore $b_{\xi}(z) \in S H_{m}(\Omega) \cap \operatorname{Lip}_{\alpha}(\bar{\Omega})$ by Definition 2.1. The other properties easily follow from the formula for $b_{\xi}(z)$. The proof is finished.

Set

$$
b(z):=\sup \left\{b_{\xi}(z): \xi \in \partial \Omega\right\} .
$$

Since $\left|b_{\xi}(z)-b_{\xi}(w)\right| \leq C|z-w|^{\alpha}, C=C(\phi, K, \rho)$, one has $b(z) \in S_{m}(\Omega) \cap \operatorname{Lip}_{\alpha}(\bar{\Omega})$. Furthermore, from properties of $b_{\xi}(z)$ we have $b(z)=\phi(z)$ on $\partial \Omega$. Hence $b(z) \leq h_{m}(z)$ in $\Omega$ by the definition of $h_{m}(z)$. 
Let $h_{1}(z)$ be the harmonic extension of $\phi$ to $\Omega$. According to Proposition 2.4 we know that $h_{1}(z) \in \operatorname{Lip}_{\alpha}(\bar{\Omega})$, and $h_{m}(z) \leq h_{1}(z)$ in $\Omega$. Altogether we have

$$
b(z) \leq h_{m}(z) \leq h_{1}(z) \text { in } \Omega, \quad b(z)=h_{m}(z)=h_{1}(z) \text { on } \partial \Omega .
$$

Having these properties, we may repeat the last part of the proof of Proposition 2.4 in order to get that $h_{m}(z) \in \operatorname{Lip}_{\alpha}(\bar{\Omega})$. The theorem follows.

Acknowledgments I am grateful to my advisor, professor Sławomir Kołodziej, who has patiently and thoroughly read the preliminary versions of this work. His remarks and enlightening suggestions helped a lot to improve the exposition of the paper. This work is supported by the International Ph.D Program " Geometry and Topology in Physical Models".

\section{References}

1. Bedford, E., Taylor, B.A.: The Dirichlet problem for a complex Monge-Ampère equation. Invent. Math. 37, 1-44 (1976)

2. Błocki, Z.: Weak solutions to the complex Hessian equation. Ann. Inst. Fourier (Grenoble) 55(5), 17351756 (2005)

3. Cegrell, U.: Pluricomplex energy. Acta Math. 180:2, 187-217 (1998)

4. Chen, X.X., Donaldson, S., Sun, S.: Kähler-Einstein Metrics on Fano Manifolds I: Approximation of Metrics with Cone Singularities, preprint. arXiv:1211.4566

5. Chinh, L.H.: Solutions to Degenerate Complex Hessian Equations. J. Math. Pures Appl. 100(6), 785-805 (2013)

6. Chinh, L.H.: Viscosity Solution to Complex Hessian Equations. J. Funct. Anal. 264(6), 1355-1379 (2013)

7. Demailly, J.P.: Potential theory in several complex variable. In: Lecture Notes. ICPAM, Nice (1989)

8. Demailly, J.-P., Dinew, S., Guedj, V., Pham, H.H., Kołodziej, S., Zeriahi, A.: Hölder Continuous Solutions to Monge-Ampère Equations. preprint. to apear in JEMS. arXiv:1112.1388v1

9. Dinew, S., Kołodziej, S. A priori estimates for the complex Hessian equations, preprint. arXiv:1112.3063 to appear in Analysis and PDE (2014)

10. Dinew, S., Kołodziej, S.: Liouville and Calabi-Yau Type Theorems for Complex Hessian Equations. preprint. arXiv:1203.3995v1

11. Dinh, T.C., Nguyen, V.A., Sibony, N.: Exponential estimates for plurisubharmonic functions and stochastic dynamics. J. Differ. Geom. 84, 465-488 (2010)

12. Eyssidieux, P., Guedj, V., Zeriahi, A.: Singular Kähler-Einstein metrics. J. Am. Math. Soc. 22, 607-639 (2009)

13. Gårding, L.: An inequality for hyperbolic polynomials. J. Math. Mech. 8, 957-965 (1959)

14. Guedj, V., Kołodziej, S., Zeriahi, A.: Hölder continuous solutions to Monge-Ampère equations. Bull. Lond. Math. Soc. 40, 1070-1080 (2008)

15. Gilbarg, D., Trudinger, N.: Elliptic partial differential equations of second order. Grundl. der Math. Wiss, vol. 244. Springer Verlag (1998)

16. Han, Q., Lin, F.: Elliptic partial differential equations. Courant Lecture Notes in Mathematics, vol. 1. New York University.

17. Hou, Z., Ma, X.-N., Wu, D.: A second order estimate for complex Hessian equations on a compact Kähler manifold. Math. Res. Lett. 17, 547-561 (2010)

18. Kołodziej, S.: The complex Monge-Ampère equation. Acta Math. 180(1), 69-117 (1998)

19. Kołodziej, S.: Hölder continuity of solutions to the complex Monge-Ampère equation with the right hand side in $L^{p}$. The case of compact Kähler manifolds. Math. Ann. 342, 379-386 (2008)

20. Li, S.-Y.: On the Dirichlet problems for symmetric function equations of the eigenvalues of the complex Hessian. Asian J. Math. 8, 87-106 (2004)

21. Nguyen, N.-C.: Subsolution Theorem for the Complex Hessian Equation. Univ. Iagiell. Acta Math. 50, 69-88 (2012)

22. Phong, D.H., Song, J., Sturm, J.: Complex Monge-Ampère equations. Surv. Differ. Geom. 17, 327-411 (2012) 